\title{
Eficacia y seguridad del hierro polimaltosado para gestantes con anemia: revisión sistemática y metaanálisis
}

\author{
Efficacy and safety of iron polymaltose in pregnant women with anemia: \\ a systematic review and meta-analysis
}

\author{
BeatrizCatherineBonilla-Untiveros ${ }^{1,2} \odot$ \\ catherinbonilla@gmail.com
}

Bárbara Rivero Cárdenas ${ }^{1,3}{ }^{\oplus}$

pcnubriv@upc.edu.pe

Artículo recibido: $31 / 05 / 2021$

Revisado por pares

Artículo aceptado: 28/01/2022

Artículo publicado: 25/02/2022

Autor de correspondencia

Barbara Rivero Cárdenas,

pcnubriv@upc.edu.pe

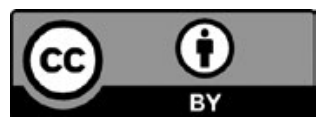

(CLas autoras, 2022. Publicado por la Universidad Norbert Wiener (Lima, Perú)

Citar como: Bonilla-Untiveros B, Rivero B. Eficacia y seguridad del hierro polimaltosado para gestantes con anemia: Revisión sistemática y metaanálisis. Revista de Investigación (de la Universidad Norbert Wiener). 2022; 11(1): r0001. doi:https://doi.org/10.37768/unw.rinv.11.01.r0001

\begin{abstract}
Resumen
Antecedentes: La anemia en gestantes es un problema de salud pública. El manejo terapéutico consiste en administrar diariamente $120 \mathrm{mg}$ de hierro elemental más ácido fólico durante 6 meses. Objetivo: Sistematizar evidencia actualizada con relación a la efectividad, seguridad y adherencia al hierro polimaltosado (HPM) en el tratamiento de anemia en gestantes, en comparación con el sulfato ferroso (SF). Método de búsqueda: Se buscaron ensayos aleatorizados y no aleatorizados en Medline (Ovid), Embase (Ovid), Cochrane Library, Lilacs y literatura gris. Recolección y análisis de datos: Dos autores seleccionaron los artículos, extrajeron los datos y realizaron verificaciones para verificar su exactitud. Se utilizó el enfoque Grade para evaluar la calidad de la evidencia para los resultados primarios. Se anticipó una alta heterogeneidad; por lo tanto, los resultados se agruparon mediante un modelo de efectos aleatorios. Resultados principales: Se incluyeron 2 ensayos clínicos. El análisis combinado de los ensayos con una duración de 8 semanas muestra un incremento significativo en los niveles de ferritina sérica con el uso del HPM (DM 6.06 IC 95\% 3,25- 8,87) (certeza de la evidencia moderada), sin encontrar diferencia significativa en los niveles de hemoglobina sérica (DM -0.03 IC 95\% -0,34-0,28) (certeza de la evidencia baja). Se observó que las gestantes con anemia que recibieron HPM presentaron menos efectos adversos y una mayor adherencia a la suplementación. Conclusión: La evidencia identificada sugiere una eficacia similar en ambos grupos; sin embargo, la tolerancia y la adherencia al HPM es mucho mejor.
\end{abstract}

Palabras clave: anemia, gestante, polimaltosado, sulfato ferroso

\begin{abstract}
Background: Anemia in pregnant women is a public health problem. Therapeutic management consists in administering $120 \mathrm{mg}$ of elemental iron and folic acid daily for six months. Objective: To systematize updated evidence regarding the effectiveness, safety and adherence to iron polymaltose (IP) in the treatment of anemia in pregnant women, compared to ferrous sulfate (FS). Search method: Randomized and non-randomized trials as well as gray literature were searched on MEDLINE (Ovid), EMBASE (Ovid), The Cochrane Library, Lilacs. Data collection and analysis: Two authors selected the articles, extracted the data and carried out checks to verify their accuracy. The GRADE approach was used to assess the quality of the evidence for the primary results. Since high heterogeneity was anticipated, the results were pooled using a random effects model. Main results: Two randomized
\end{abstract}

\footnotetext{
${ }^{1}$ Unidad de Análisis y Generación de Evidencias en Salud Pública, Instituto Nacional de Salud. Lima, Perú.

${ }^{2}$ Escuela Profesional de Nutrición, Facultad de Medicina, Universidad Nacional Mayor de San Marcos. Lima, Perú.

${ }^{3}$ Escuela de Nutrición y Dietética, Facultad de Ciencias de la Salud, Universidad Peruana de Ciencias Aplicadas. Lima, Perú.
} 
controlled studies were included. The combined analysis of the trials, which lasted eight weeks, shows a significant increase in serum ferritin levels with the use of IP (MD 6.06 95\% CI 3.25, 8.87) (moderate certainty of evidence), without finding a significant difference in serum hemoglobin levels (MD $-0.0395 \%$ CI $-0.34,0.28$ ) (low certainty of the evidence). It was observed that pregnant women with anemia who received IP had fewer adverse effects and greater adherence to supplementation. Conclusion: The identified evidence suggests a similar efficacy in both groups. However, tolerance and adherence to IP is much better.

Keywords: anemia, pregnant women, polymaltose, ferrous sulfate

\section{INTRODUCCIÓN}

La anemia es una condición que afecta principalmente a mujeres gestantes, en edad fértil y a niños menores de cinco años. La anemia por deficiencia de hierro es la más prevalente y ocasiona desventajas a nivel cognitivo, económico, de salud, entre otros $\left({ }^{1}\right)$. En las gestantes con dicha condición, existe un mayor riesgo de dar a luz un neonato con bajo peso o prematuro, y aumenta el riesgo de mortalidad perinatal y neonatal $\left({ }^{2}\right)$.

La anemia durante la gestación es diagnosticada cuando la hemoglobina es menor a $11 \mathrm{~g} / \mathrm{dl}$ en zonas geográficas ubicadas por debajo de los $1000 \mathrm{~m} \mathrm{s.n.m.}$ Sin embargo, en el segundo trimestre del embarazo, entre las semanas 13 y 28 , el diagnóstico de anemia se realiza cuando los valores de hemoglobina están por debajo de $10,5 \mathrm{~g} / \mathrm{d}\left({ }^{3}\right)$. En el mundo, se calcula que un $38,2 \%$ de las gestantes padece anemia $\left({ }^{3}\right)$. En el Perú, según datos de la Encuesta Demográfica y Salud Familiar (Endes) 2019, el 28,2\% de las embarazadas encuestadas presentó algún grado de anemia $\left({ }^{4}\right)$. Asimismo, se ha documentado que la prevalencia es mayor $(>32 \%)$ en las regiones Huancavelica, Pasco, Cusco y Apurímac $\left({ }^{5}\right)$.

En el Perú, la norma técnica Manejo Terapéutico y preventivo de la anemia en niños, adolescentes, mujeres gestantes y puérperas establece que el manejo terapéutico de la gestante consiste en administrar diariamente $120 \mathrm{mg}$ de hierro elemental más $800 \mu \mathrm{g}$ de ácido fólico durante 6 meses, y considera el uso tanto de sulfato ferroso (SF) como de hierro polimaltosado (HPM); además, indica que, en caso exista una inadecuada adherencia $(<75 \%)$ al consumo del SF, se debe optar por el HPM $\left(^{6}\right)$. Esto debido a que el HPM es un complejo de liberación lenta. La polimaltosa actúa como una envoltura del hierro, lo cual asegura que este se libere lentamente. En consecuencia, produce menos efectos secundarios en comparación con otros suplementos y favorece así la adherencia al tratamiento.

Se sabe que la adherencia al tratamiento es fundamental para conseguir una mejora en la hemoglobina y otros indicadores bioquímicos $\left({ }^{7}\right)$. Algunos estudios realizados en el Perú evidenciaron que los efectos adversos, como el estreñimiento, la cefalea y las náuseas, entre otros, juegan un rol importante en la adherencia a la suplementación con SF $(7,8)$. En tal sentido, resulta relevante que las recomendaciones de los profesionales de la salud se basen en estudios con diseños adecuados que evalúen su efectividad y seguridad, de manera que se pueda elegir el suplemento más idóneo para tratar la anemia en gestantes.

Resulta de interés evaluar la eficacia y la seguridad comparativa entre las opciones terapéuticas en nuestro país, como tratamientos de primera línea, para identificar el balance riesgo-beneficio de iniciar el tratamiento para la anemia de ambas formulaciones. Por lo tanto, el presente estudio tiene como objetivo identificar y sistematizar evidencia actualizada con relación a la efectividad, la seguridad y la adherencia al hierro polimaltosado en el tratamiento de anemia en gestantes, en comparación con el tratamiento estándar en el Perú: el sulfato ferroso.

\section{MATERIAL Y MÉTODO}

La presente revisión ha seguido la guía para reportar revisiones sistemáticas de "Preferred Reporting Items for Systematic reviews and Meta-Analysis" (Prisma) $\left.{ }^{9}\right)$, y ha sido elaborada en el marco de 
la elaboración de una Guía de práctica clínica nacional para el manejo de anemia por deficiencia de hierro. Por lo que la pregunta PICO, los criterios de inclusión y exclusión de la evidencia a buscar, no se han publicado en un protocolo propiamente dicho, sin embargo, se han redactado en la versión metodológica de la GPC, y están disponibles mediante solicitud dirigida a los autores.

La pregunta PICO de la revisión es la siguiente: ¿en gestantes con anemia, el uso hierro polimaltosado comparado con el sulfato ferroso es eficaz y seguro para su tratamiento?

La búsqueda sistemática de artículos científicos se llevó a cabo en Medline (Ovid), Embase (Ovid), Cochrane Library y Lilacs, hasta el 30 de enero de 2021, y fue realizada por un solo autor. Además, se revisó literatura gris en el Repositorio Nacional Digital de Ciencia, Tecnología e Innovación, denominado Alicia (Acceso Libre a la Información Científica) y en la página de la Organización Panamericana de la Salud (OPS). No se realizaron restricciones de idioma, año de publicación o diseño del estudio. La estrategia de búsqueda incluyó los términos "pregnant woman", "iron polymaltose", "ferrous sulfate" y "anemia".

Los criterios de inclusión de esta revisión fueron los siguientes:

- Las intervenciones fueron de ensayos aleatorizados y no aleatorizados.

- Los participantes de los estudios son gestantes con anemia, de acuerdo con la definición de cada autor.

- Las intervenciones incluyen uso de HPM, en comparación con el SF.

- Los desenlaces son aquellos relacionados con la medición de anemia.

Los criterios de exclusión se señalan a continuación:

- Estudios que incluyen gestantes con anemia y sin anemia, sin hacer una distinción entre los subgrupos

- Estudios de los que no se puede acceder al texto completo
Para seleccionar los artículos, los autores realizaron primero una lectura de títulos y resúmenes, y seguidamente la lectura a texto completo de las referencias incluidas previamente. Estos pasos se llevaron a cabo de manera independiente. Cada artículo fue leído por ambos revisores. El proceso de selección de estudios está representado en el flujograma de acuerdo con lo recomendado por Prisma (figura 1).

La extracción de información relevante fue realizada de manera independiente e incluyó los siguientes ítems: país, características de los participantes, metodología del estudio y resultados relacionados con la eficacia y la seguridad del tratamiento de anemia en gestantes.

Se realizó un metaanálisis de los desenlaces críticos, utilizando Review Manager Software versión 5.4 (10). Para las variables dicotómicas, los resultados fueron presentados como risk ratio (RR) y odds ratio (OR), y su respectivo intervalo de confianza al 95\% (IC). Para variables continuas, se utilizó la diferencia de medias si los resultados eran comparables. La heterogeneidad entre los ensayos fue evaluada utilizando el estadístico I2 $($ I2 $>30)$ y un p-valor $<0,1$ (en chi-cuadrado), y por inspección visual del forest plot. Se utilizó el modelo de efectos aleatorios.

El riesgo de sesgo de los estudios incluidos fue realizado de manera independiente hasta alcanzar el consenso, para lo que se utilizó el instrumento de evaluación de riesgo de sesgo de la Colaboración Cochrane $\left({ }^{11}\right)$. Los dominios evaluados fueron los siguientes: generación aleatoria de la secuencia, ocultación de la asignación, cegamiento de los participantes y del personal, cegamiento de los evaluadores de los resultados, datos de resultados incompletos, notificación selectiva de los resultados y otros sesgos. Fueron evaluados como "riesgo bajo", "riesgo alto" o "riesgo poco claro" de sesgo.

La evidencia fue presentada según desenlaces y, para cada desenlace, se valoró la calidad de la evidencia de manera independiente por los dos autores, utilizando el enfoque Grading of Recommendations Assessment, Development and Evaluation (Grade), el cual considera la valoración de riesgo de sesgo de cada estudio, la evidencia indirecta, la heterogeneidad, la precisión del efecto 
estimado y el riesgo de sesgo de publicación. La valoración de la certeza de evidencia se gradúa en alto, moderado, bajo o muy bajo $\left({ }^{12}\right)$.

\section{RESULTADOS}

La búsqueda sistemática identificó 25 artículos científicos. Luego de la lectura por título y resumen, se seleccionaron 4 estudios para la lectura a texto completo. De estos estudios, se incluyeron dos ensayos clínicos $\left({ }^{13}, 14\right)$. Se excluyeron dos estudios, una evaluación de tecnología sanitaria $\left({ }^{15}\right)$ que incluía un artículo seleccionado previamente y una revisión sistemática $\left.{ }^{(16}\right)$. Esta revisión tuvo como objetivo revisar la efectividad de todas las intervenciones para el tratamiento de anemia en gestantes. Al realizar la comparación del HPM con el SF, solo incluye el análisis un estudio seleccionado por nuestra revisión $\left({ }^{14}\right)$ (figura 1 ).

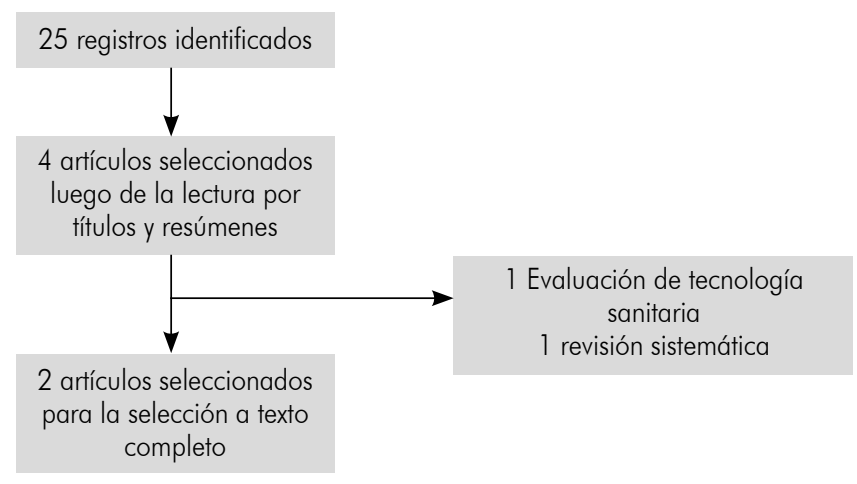

Figura 1. Diagrama de flujo Prisma del proceso de selección

\section{a. Características de los estudios incluidos}

Saha et al. $\left({ }^{14}\right)$ desarrollaron un ensayo clínico doble ciego, en India, para comparar la eficacia y seguridad del SF (SF) y el HPM (HPM) para el tratamiento de la anemia en gestantes, entre septiembre 2003 y octubre 2004. Las gestantes tenían que contar con un diagnóstico bioquímico de anemia por deficiencia de hierro y no debían presentar historia de otras causas de anemia. La edad promedio de las gestantes era de 27 años, se encontraban entre la semana 14 y 27 de gestación, y ambos grupos presentaban anemia moderada $(8,39 \pm 0,74$ en el grupo de SF y 8,47 $\pm 0,72$ en el grupo de HPM). Del total de 100 gestantes del estudio, 48 recibieron HPM (100 mg de hierro elemental + 500 mcg de ácido fólico, 1 tableta al día) y 52 SF (120 mg de hierro + 500 mcg de ácido fólico, cada tableta de 60 mg administrada dos veces al día). Ambos grupos fueron seguidos por ocho semanas. Los desenlaces evaluados fueron los siguientes: hemoglobina, anemia, otros indicadores bioquímicos, eventos adversos, adherencia al tratamiento y costos de tratamiento.

Ortiz et al. $\left({ }^{13}\right)$, mediante un ensayo clínico multicéntrico realizado en dos países latinoamericanos (Colombia y Argentina), buscaron comparar la eficacia y la seguridad entre el SF y el HPM para el tratamiento de anemia en gestantes. El estudio se realizó entre diciembre 2005 y julio 2006. Los criterios de selección para las gestantes fueron estos: edad de 16 años a más, encontrarse entre las semanas 18 y 26 de gestación, y contar con indicadores bioquímicos de anemia por deficiencia de hierro; además, no debían presentar sobrecarga de hierro, alteraciones en la utilización de hierro, anemia debida a otras causas $u$ otras enfermedades clínicas importantes. La edad promedio en ambos grupos fue de 27 años, edad gestacional entre 13 y 25 semanas, y los valores promedio de $\mathrm{Hb}$ las clasifica como gestantes con anemia moderada $(9,64 \pm 0,89$ en el grupo de HPM y 9,79 $\pm 0,64$ en el grupo de SF). Ochenta mujeres fueron aleatorizadas: 39 mujeres recibieron SF y 41 al grupo de HPM, y la dosis en ambos grupos fue de $200 \mathrm{mg}$ de hierro al día, durante o después de las comidas. Cada país tuvo distintas presentaciones de HPM: cápsulas en envases de dosis únicas en Colombia o comprimido recubierto con película en Argentina.

\section{b. Evaluación del riesgo de sesgo}

El método utilizado para la generación aleatoria de la secuencia no es claro en ninguno de los estudios. Solo el de Ortiz et al. describió utilizar un método para ocultar la asignación aleatoria. Mientras que el estudio de Saha et al. consideró el cegamiento de los participantes y los profesionales de salud. Respecto del cegamiento de los evaluadores de resultados, ningún estudio lo explicita. En cuanto a los dominios de datos de resultados incompletos y la notificación selectiva de resultados, ambos estudios obtuvieron una valoración de bajo riesgo de sesgo. Por último, el estudio Ortiz et al. obtuvo una valoración de alto riesgo de sesgo en el dominio de 
Tabla 1. Resumen de las características de los estudios incluidos

\begin{tabular}{|c|c|c|}
\hline Primer autor, año de publicación & Saha et al., 2007 & Ortiz et al., 2011 \\
\hline Lugar del estudio & India & Colombia y Argentina \\
\hline Características de los participantes & $\begin{array}{l}\text { Edad promedio: } \\
\text { HPM: } 27,58 \pm 4,2 \\
\text { SF: } 27,54 \pm 3,5 \\
\text { Edad gestacional media al inicio: } \\
\text { HPM: } 18,58 \pm 2,8 \\
\text { SF } 17,62 \pm 3,1 \\
\text { Hb basal: } \\
\text { HPM: } 8,47 \pm 0,72 \\
\text { SF: } 8,39 \pm 0,74\end{array}$ & $\begin{array}{l}\text { Edad promedio: } \\
\text { HPM: } 26,8 \pm 6,2 \\
\text { SF: } 26,8 \pm 6,2 \\
\text { Edad gestacional media al inicio: } \\
\text { HPM: } 20,1 \pm 2,7 \\
\text { SF19,6 } 2 \pm 2,3 \\
\text { Hb basal: } \\
\text { HPM: } 9,64 \pm 0,89 \\
\text { SF: } 9,79 \pm 0,64\end{array}$ \\
\hline $\begin{array}{l}\text { Número de participantes } \\
\text { (Grupo HPM/Grupo SF) }\end{array}$ & $100(48 / 52)$ & $80(41 / 39)$ \\
\hline Duración del estudio & 8 semanas & 90 días ( $\approx 12$ semanas) \\
\hline Suplementación: grupo HPM & $\begin{array}{l}100 \mathrm{mg} \text { de hierro elemental }+500 \mathrm{mcg} \text { de } \\
\text { ácido fólico por ( } 1 \text { tableta oral, una vez al día) }\end{array}$ & $\begin{array}{l}200 \mathrm{mg} \text { de hierro elemental (100 mg dos veces } \\
\text { al día). Dos presentaciones distintas por cada } \\
\text { país }\end{array}$ \\
\hline Suplementación: grupo SF & $\begin{array}{l}60 \mathrm{mg} \text { de hierro elemental }+500 \mathrm{mcg} \text { de ácido } \\
\text { fólico por ( } 1 \text { tableta oral, dos veces al día) }\end{array}$ & $\begin{array}{l}200 \mathrm{mg} \text { de hierro elemental (100 mg dos veces } \\
\text { al día) }\end{array}$ \\
\hline Principales desenlaces evaluados & $\begin{array}{l}\text { Prevalencia de gestantes que alcancen un nivel } \\
\text { de hemoglobina }>11 \mathrm{~g} / \mathrm{dl} \text {. } \\
\text { Hemoglobina y efectos adversos medidos en la } \\
\text { semana } 0,2,4,6 \text { y } 8 \text {. } \\
\text { Hematocrito, volumen corpuscular medio } \\
\text { (VCM), hemoglobina corpuscular media (HCM), } \\
\text { concentración de hemoglobina corpuscular } \\
\text { media (CHCM), hierro sérico, ferritina sérica; } \\
\text { medidos en la primera y octava semana. }\end{array}$ & $\begin{array}{l}\text { Hemoglobina, ferritina sérica, saturación de } \\
\text { transferrina, hierro sérico, hematocrito, volumen } \\
\text { corpuscular medio (VCM), hemoglobina } \\
\text { corpuscular media (HCM) y concentración } \\
\text { de hemoglobina corpuscular media (CHCM); } \\
\text { medidos en los días } 0,30,60 \text { y } 90 \text {. } \\
\text { Efectos adversos }\end{array}$ \\
\hline
\end{tabular}

otras fuentes de sesgo, debido a que fue financiado por el laboratorio que produjo el suplemento de HPM brindado en el estudio, y dos de los autores declararon haber recibido financiamiento por el laboratorio en otras ocasiones.

\section{c. Resultados relacionados con eficacia y/o efectividad}

En el estudio de Saha et al. se observa en todas las gestantes un incremento significativo en los niveles de $\mathrm{Hb}$ después de las 8 semanas (incremento promedio \pm DS de $2,9 \pm 0,46 \mathrm{~g} / \mathrm{dL}$ en grupo SF y $2,72 \pm 0,55 \mathrm{~g} / \mathrm{dL}$ en el grupo de HPM), pero no encuentra diferencias al comparar entre ambos grupos $(p=0,07)$. Estos incrementos se observan desde la primera evaluación en la semana 4 , y en las mediciones siguientes en las semanas 6 y 8 ; todos estos incrementos fueron estadísticamente significativos comparado con los valores iniciales. La tasa de recuperación de anemia (alcanzar una $\mathrm{Hb}$ $>11 \mathrm{~g} / \mathrm{dL})$ fue del $63,5 \%(33 / 52)$ y el $68,8 \%(33 / 48)$ en los grupos de SF y de HPM, respectivamente.
Además, se observaron incrementos significativos en todas las gestantes en los valores finales de hematocrito, volumen corpuscular medio (VCM), hemoglobina corpuscular media (HCM), concentración de hemoglobina corpuscular media (CHCM), hierro sérico y ferritina sérica. $\mathrm{Al}$ comparar estos indicadores entre ambos grupos, solo se observó diferencia estadísticamente significativa en los valores de ferritina sérica, siendo mayor el incremento en el grupo de HPM $(22,59 \pm$ $6,8 \mathrm{ng} / \mathrm{mL})$ comparado con el grupo de SF $(16,84 \pm$ $7,9 \mathrm{ng} / \mathrm{mL})\left({ }^{14}\right)$.

Por otro lado, el estudio de Ortiz et al. observó que las gestantes en ambos grupos incrementaron de manera significativa de los valores de $\mathrm{Hb}$ al día 90 (incremento promedio \pm DS $1,93 \pm 0,97 \mathrm{~g} / \mathrm{dL}$ en SF y 2,16 $\pm 0,67 \mathrm{~g} / \mathrm{dL}$ en HPM), y tampoco encontró diferencias entrelas dosintervenciones $(\mathrm{p}=0,25)$. En la medición final todas las gestantes se recuperaron de la anemia (promedio 11,89 g/dL con HPM y $11,70 \mathrm{~g} / \mathrm{dL}$ con SF; $\mathrm{p}=0,056)$. Al comparar los parámetros hematológicos con el basal, se observó 
que hematocrito, saturación de transferrina, hierro sérico y ferritina sérica presentaron incrementos significativos en las gestantes que consumieron HPM. Al comparar estos resultados con el grupo que recibió SF, el incremento de ferritina sérica, fue significativamente mayor en el grupo de HPM (incremento promedio $64 \pm 40 \mathrm{ng} / \mathrm{mL}$ con HPM y $41 \pm 28 \mathrm{ng} / \mathrm{mL}$ con SF; $\mathrm{p}=0,004)\left({ }^{13}\right)$.

El análisis combinado de los ensayos con una duración de 8 semanas muestra un incremento significativo en los niveles de ferritina sérica con el uso del HPM (DM 6,06 IC 95\% 3,25-8,87) (certeza de la evidencia moderada), sin encontrar diferencia significativa en los niveles de hemoglobina sérica
(DM -0,03 IC 95\% $-0,34$ a 0,28) (certeza de la evidencia baja) (figuras 2 y 3 ).

La certeza de evidencia para el desenlace de hemoglobina fue calificada como moderada, mientras que la certeza de la evidencia para el desenlace de ferritina sérica fue valorado como moderada (tabla 2).

\section{d. Resultados relacionados con la seguridad}

Según Saha et al., las gestantes reportaron mayores efectos con SF (78\%, 41/52 pacientes) que con el HPM (31\%, $15 / 48$ pacientes), diferencia estadísticamente significativa $(p<0,001)$. Los

\begin{tabular}{|c|c|c|c|c|c|c|c|c|c|c|}
\hline Study or Subgroup & \multicolumn{2}{|c|}{ Experimental } & $\begin{array}{l}\text { tal } \\
\text { Total }\end{array}$ & \multicolumn{3}{|c|}{ Control } & Weight & $\begin{array}{l}\text { Mean Difference } \\
\text { IV, Random, 95\% CI }\end{array}$ & \multicolumn{2}{|c|}{$\begin{array}{c}\text { Mean Difference } \\
\text { IV, Random, } 95 \% \mathrm{CI}\end{array}$} \\
\hline Saha 2007 & 22.59 & 6.8 & 48 & 16.84 & 7.9 & 52 & $95.0 \%$ & $5.75[2.87,8.63]$ & & -- \\
\hline Ortiz 2011 & 32 & 26 & 41 & 20 & 31 & 39 & $5.0 \%$ & $12.00[-0.57,24.57]$ & & \\
\hline Total $(95 \% \mathrm{CI})$ & & & 89 & & & 91 & $100.0 \%$ & $6.06[3.25,8.87]$ & & \\
\hline $\begin{array}{l}\text { Heterogeneity. Tau } \\
\text { Test for overall effec }\end{array}$ & $\begin{array}{l}0.00 ; C \\
Z=4.2\end{array}$ & $=$ & $\begin{array}{l}0.90, d \\
0.000\end{array}$ & $=10$ & $=0$ & $34) ; 1^{2}=$ & $=0 \%$ & & $\begin{array}{ll}-10 & -10 \\
\text { Favours [control] }\end{array}$ & $\begin{array}{ccc}1 & 10 & 20 \\
\text { Favours [experimental] }\end{array}$ \\
\hline
\end{tabular}

Los resultados están presentados en diferencia de medias (DM ng/mL, con IC-95\%)

Figura 2. Efecto del HPM comparado con SF en los niveles de ferritina sérica en gestantes con anemia

\begin{tabular}{|c|c|c|c|c|c|c|c|c|c|c|}
\hline \multirow[b]{2}{*}{ Study or Subgroup } & \multicolumn{3}{|c|}{ Experimental } & \multicolumn{3}{|c|}{ Control } & \multicolumn{2}{|c|}{ Mean Difference } & \multirow{2}{*}{\multicolumn{2}{|c|}{$\begin{array}{c}\text { Mean Difference } \\
\text { IV, Random, 95\% CI }\end{array}$}} \\
\hline & Mean & SD & Total & Mean & SD & Total & Weight & IV, Random, 95\% CI & & \\
\hline Ortiz 2011 & 1.41 & 0.38 & 41 & 1.27 & 0.73 & 39 & $46.7 \%$ & $0.14[-0.12,0.40]$ & $f$ - & \\
\hline Saha 2007 & 2.72 & 0.55 & 48 & 2.9 & 0.46 & 52 & $53.3 \%$ & $-0.18[-0.38,0.02]$ & & \\
\hline Total $(95 \% \mathrm{Cl})$ & & & 89 & & & 91 & $100.0 \%$ & $-0.03[-0.34,0.28]$ & & \\
\hline $\begin{array}{l}\text { Heterogeneity. Tau }{ }^{2} \\
\text { Test for overall effect }\end{array}$ & $\begin{array}{l}0.04 ; \\
z=0.1\end{array}$ & $\begin{array}{l}\mathrm{hi}^{2}=3 \\
9(\mathrm{P}=\end{array}$ & $\begin{array}{l}3.72, d \\
0.851\end{array}$ & $=1$ & $=0$. & $; 1^{2}=$ & $=73 \%$ & & $\begin{array}{ccc}-1 & 1 \\
-2 & -1 \\
& \text { Favours [control] }\end{array}$ & rimental] \\
\hline
\end{tabular}

Los resultados están presentados en diferencia de medias (DM g/dL, con IC-95\%)

Figura 3: Efecto del HPM comparado con SF en los niveles de hemoglobina en gestantes con anemia

Tabla 2. Perfil de evidencia Grade

\begin{tabular}{|c|c|c|c|c|c|c|c|c|c|c|c|c|}
\hline \multicolumn{7}{|c|}{ Evaluación de certeza } & \multicolumn{2}{|c|}{$\begin{array}{c}\mathrm{N}^{\circ} \text { de } \\
\text { pacientes }\end{array}$} & \multicolumn{2}{|c|}{ Efecto } & \multirow[t]{2}{*}{ Certeza } & \multirow[t]{2}{*}{ Importancio } \\
\hline $\begin{array}{l}N^{\circ} \text { de } \\
\text { estudios }\end{array}$ & $\begin{array}{c}\text { Diseño de } \\
\text { estudio }\end{array}$ & $\begin{array}{c}\text { Riesgo } \\
\text { de sesgo }\end{array}$ & Inconsistencia & $\begin{array}{l}\text { Evidencia } \\
\text { indirecta }\end{array}$ & Imprecisión & $\begin{array}{c}\text { Otras } \\
\text { consideraciones }\end{array}$ & HPM & SF & $\begin{array}{c}\text { Relativo } \\
(95 \% \mathrm{Cl}) \\
\end{array}$ & $\begin{array}{l}\text { Absoluto } \\
(95 \% \mathrm{CI})\end{array}$ & & \\
\hline \multicolumn{13}{|c|}{ Hemoglobina (seguimiento de 8 semanas; evaluado con g/dL) } \\
\hline 2 & $\begin{array}{l}\text { ensayos } \\
\text { aleatorios }\end{array}$ & serio a & serio $b$ & $\begin{array}{l}\text { no es } \\
\text { serio }\end{array}$ & no es serio & ninguno & 89 & 91 & - & $\begin{array}{c}0,03 \text { menor } \\
(0,34 \text { menor } \\
\text { a } 0,28 \text { más } \\
\text { alto) }\end{array}$ & $\underset{\text { baja }}{\oplus \oplus \bigcirc \bigcirc}$ & crítico \\
\hline \multicolumn{13}{|c|}{ Ferritina sérica (seguimiento de 8 semanas; evaluado con $\mathrm{ng} / \mathrm{dL}$ ) } \\
\hline 2 & $\begin{array}{l}\text { ensayos } \\
\text { aleatorios }\end{array}$ & serio a & no es serio c & $\begin{array}{l}\text { no es } \\
\text { serio }\end{array}$ & no es serio & ninguno & 89 & 91 & - & $\begin{array}{l}\text { 6,06 más } \\
\text { alto (3,25 } \\
\text { más alto. a } \\
8,87 \text { más } \\
\text { alto) }\end{array}$ & $\begin{array}{l}\oplus \oplus \oplus \bigcirc \\
\text { moderado }\end{array}$ & crítico \\
\hline
\end{tabular}


principales eventos adversos reportados fueron problemas gastrointestinales (gastritis, acidez) y estreñimiento, además de sabor metálico, diarrea y sarpullido, reportados con menor frecuencia $\left({ }^{14}\right)$.

El estudio de Ortiz et al. utilizó el doble de la dosis que el estudio de Saha et al. y reportó 34 mujeres con eventos adversos: 12/41 (29,3\%) en el grupo de HPM y 22/39 (56,4\%) en el grupo de SF $(\mathrm{p}=0,015)$, asociados principalmente con molestias gastrointestinales (náusea, vómitos $\mathrm{y}$ estreñimiento).

\section{e. Resultados relacionados con la adherencia}

Para medir la adherencia al tratamiento, en el estudio de Saha et al. se contaron las tabletas en cada visita. La adherencia fue alta en ambos grupos $(80 \%)$ y significativamente mayor $(\mathrm{p}<0,05)$ en el grupo de HP, comparado con el grupo de SF $(90 \%$ vs. $87 \%$, respectivamente).

Ortizetal.realizaronla comparación delaadherencia entre ambos grupos con el conteo de tabletas que regresaban en cada visita, y consideraron como favorable cuando no regresaba ninguna tableta en la visita. En ese sentido, del $41-46 \%$ de gestantes en el grupo de SF retornó cero tabletas, mientras que en el grupo de HPM, el porcentaje fue de $66-73 \%$.

\section{DISCUSIÓN}

La evidencia sugiere que no existe diferencia entre el HPM y el SF para el tratamiento de la anemia en gestantes. Los estudios identificados presentan incrementos similares en los niveles de hemoglobina, luego de 8 semanas de intervención. Al comparar los valores, se observa que el incremento fue mayor para ambos grupos en el estudio de Saha et al., en comparación con el estudio de Ortiz et al., a pesar de que este último administró una dosis superior y por más tiempo. Existe evidencia que difiere con estos hallazgos, pues se ha visto que existe una relación dosis-respuesta positiva entre la cantidad de hierro suministrada y los cambios en los valores de hemoglobina en gestantes $\left({ }^{17}\right)$. Adicionalmente, un estudio realizado en donantes de sangre con deficiencia de hierro demostró mayor eficacia al proporcionar $200 \mathrm{mg}$ de HPM en comparación con suministrar solo $100 \mathrm{mg}\left({ }^{18}\right)$. Es interesante agregar que los valores basales de $\mathrm{Hb}$ en las gestantes con mayor respuesta fueron más bajos comparados con aquellas en el estudio de Ortiz et al.

La evidencia analizada indica que es probable que el consumo de HPM en gestantes con diagnóstico de anemia por un periodo de 8 semanas incremente los niveles de ferritina sérica. El incremento observado en los grupos que recibieron HPM es estadísticamente mayor que el observado con el SF. La mayoría de las gestantes con anemia presentan una depleción importante de los depósitos de hierro $y$, considerando que la ferritina sérica es una proteína de almacenamiento de hierro, dicho indicador tendría una respuesta más rápida en comparación con los valores de hemoglobina. Se ha evidenciado que la ferritina sérica, además de la hemoglobina, es un indicador de suma relevancia para el seguimiento de una población con anemia, considerando que la principal causa es la deficiencia de hierro; por lo que debemos prestar atención a dichos resultados $\left({ }^{19}\right)$.

Adicionalmente, no se encontraron diferencias al observar la mayoría de los indicadores hematológicos. Ello pudo deberse a la corta duración de la suplementación, 8 semanas en el estudio de Saha et al. y alrededor de 12 semanas en el estudio de Ortiz et al.; cuando la mayoría de estudios que evaluaron el efecto de la suplementación en gestantes tuvo una duración aproximada de 17 semanas $\left({ }^{20}\right)$.

A la fecha, la evidencia encontrada para comparar los hallazgos de eficacia entre el HPM y SF en gestantes con anemia comprende una revisión sistemática y una evaluación de tecnología sanitaria. Como se mencionó anteriormente, estos identificaron los dos estudios seleccionados en la presente revisión $\left({ }^{15,16}\right)$. En tanto, una revisión que evaluó la eficacia del HPM comparándolo con SF, en población adulta, encontró que ambos grupos alcanzaron niveles de hemoglobina semejantes, lo que sugiere una eficacia similar $\left({ }^{21}\right)$.

Respecto de los resultados relacionados con la seguridad, se encontró que los eventos adversos fueron más frecuentes en el grupo de SF. Este hallazgo coincide con lo reportado por Melamed et al., un estudio observacional que da a conocer los eventos adversos para distintos tipos de suplemento 
de hierro a partir del autorreporte de las gestantes ${ }^{(22}$. En el estudio se pudo evidenciar que 29 de 54 de gestantes $(53,7 \%)$ reportaron algún evento adverso luego de consumir SF de liberación inmediata durante el segundo y tercer trimestre. Mientras que 22 de 56 gestantes $(39,3 \%)$ reportaron algún efecto luego de consumir HPM. Estos resultados son más parecidos a los reportados en el estudio de Ortiz et al. Es probable que la recomendación de consumir el suplemento en horarios alrededor de las comidas, podría haber influenciado en la menor cantidad de eventos adversos reportados, en comparación con el estudio de Saha et al., considerando que la dosis consumida fue mayor en el primer estudio.

Los efectos adversos juegan un rol importante en la adherencia a la suplementación del hierro. Algunos estudios reportan que la curva de adherencia muestra una tendencia a la baja, mientras que los eventos adversos revelan una tendencia creciente, a medida que aumenta la dosis de SF $\left({ }^{23}\right)$. Una revisión sistemática y metaanálisis estimó que la adherencia a la suplementación con hierro y ácido fólico en mujeres embarazadas en Etiopía alcanzaba apenas un $46,15 \%\left({ }^{24}\right)$.

Dicho resultado coincide con lo reportado por Ortiz, quien encontró una adherencia entre el $41-46 \%$ en las gestantes que recibieron SF, pero difiere con los resultados del estudio de Saha et al., cuya adherencia reportada está por encima del $80 \%$. Esta mayor adherencia al tratamiento con hierro en gestantes podría deberse al conocimiento de la gestante sobre los riesgos de anemia. Una revisión sistemática estimó que el tener una complicación de la anemia durante el embarazo se asocia positivamente con la adherencia a la suplementación con hierro $\left({ }^{25}\right)$.

Nuestros resultados permiten recomendar ambas presentaciones de hierro para el manejo de anemia en gestantes. Sin embargo, considerando los efectos sobre el nivel de ferritina sérica, los efectos adversos y la adherencia al suplemento son cruciales para una la pronta recuperación de la gestante, se observan mayores beneficios con el uso de HPM frente al SF. La normativa peruana actual específica que en caso la adherencia sea menor del 75\% al SF, debe cambiar a HPM. Nuestros resultados permiten sugerir que el HPM puede ser utilizado como tratamiento de primera línea en gestantes con anemia.
En nuestro país, la elección del SF como primeralínea de tratamiento, podría deberse a la diferencia entre los precios de dichos suplementos. Sin embargo, el estudio de Saha et al. demostró que, si bien existe diferencia significativa en el costo del suplemento per se, al adicionar costos indirectos producto de los efectos adversos, la diferencia dejaba de ser significativa. Valdría la pena, además, reflexionar sobre el miedo o rechazo que podría generar en la gestante al no obtener buenos resultados tras recibir un primer suplemento hierro, pues se sabe que el miedo es una de las principales razones de la baja adherencia a la suplementación en gestantes $\left({ }^{24}\right)$.

En el presente estudio solo se han identificado dos estudios, cuyos resultados deben ser revisados e interpretados con cuidado dada la evaluación de riesgo de sesgo. Si bien el estudio de Saha et al. presenta menor riesgo de sesgo en la mayoría de los criterios, el estudio de Ortiz et al. presenta alto riesgo de sesgo en más de un dominio. En ese sentido, sería necesario realizar más estudios controlados aleatorizados de calidad para valorar la eficacia y seguridad del HPM en gestantes con y sin anemia bajo diferentes esquemas de tratamiento. Adicionalmente, subyace la necesidad de evaluar la costo-efectividad del HPM en comparación al SF, así como evaluar las barreras y facilitadores de la adherencia a la suplementación con HPM en gestantes.

\section{CONCLUSIONES}

El presente estudio evaluó la eficacia, la seguridad y la adherencia del hierro polimaltosa en comparación con el sulfato ferroso en gestantes con anemia. La evidencia identificada sugiere que el incremento de hemoglobina en gestantes con anemia podría ser similar utilizando el SF o el HPM. Sin embargo, el tratamiento con HPM permite un mayor incremento en los valores de ferritina sérica en comparación con quienes consumen SF. Además, las gestantes con anemia que recibieron HPM presentaron menos efectos adversos y una mayor adherencia a la suplementación. Se necesita más investigación en el tema para validar los hallazgos del presente estudio. 


\section{CONTRIBUCIÓN DE AUTORÍA}

Catherine Bonilla-Untiveros y Bárbara Rivero elaboraron la pregunta de investigación, realizaron la búsqueda, la selección de estudios y la evaluación de riesgo de sesgo. CB realizó los análisis estadísticos (metaanálisis). La redacción de la versión final fue elaborada por ambas autoras.

\section{REFERENCIAS BIBLIOGRÁFICAS}

1. World Health Organization. Iron deficiency anaemia assessment, prevention, and control: a guide for programme managers. 2011. Disponible en: http:// apps.who.int/iris/bitstream/10665/66914/1/WHO_ NHD_01.3.pdf?ua=1

2. Rahman MM, Abe SK, Rahman MS, Kanda M, Narita S, Bilano V, et al. Maternal anemia and risk of adverse birth and health outcomes in low- and middle-income countries: systematic review and meta-analysis. Am J Clin Nutr. 2016; 103(2): 495504.

3. World Health Organization. The global prevalence of anaemia in 2011 [Internet]. 2015 [citado el 5 de mayo de 2021]. Disponible en: http://www.who. int/entity/nutrition/publications/micronutrients/ global_prevalence_anaemia_2011/en/index.html

4. Instituto Nacional de Estadística e Informática. Encuesta Demográfica y de Salud Familiar Endes 2019. 2018. Disponible en: https://www.inei.gob. pe/media/MenuRecursivo/publicaciones_digitales/ Est/Endes2019/

5. Hernández-Vásquez A, Azañedo D, Antiporta DA, Cortés S. Análisis espacial de la anemia gestacional en el Perú, 2015. Rev Peru Med Exp Salud Pública. 2017; 34: 43-51.

6. Ministerio de Salud del Perú. Norma Técnica Manejo Terapéutico y preventivo de la anemia en niños, adolescentes, mujeres gestantes y puérperas. 2017. Disponible en: http://bvs.minsa.gob.pe/local/ MINSA/4190.pdf

\section{POTENCIALES CONFLICTOS DE INTERESES}

Ninguno.

\section{FINANCIAMIENTO}

Esta revisión sistemática se realizó con el financiamiento del Instituto Nacional de Salud del Perú.
7. Zavaleta N, Caulfield LE, Figueroa A, Chen P. Patterns of compliance with prenatal iron supplementation among Peruvian women. Matern Child Nutr. 2014; 10(2): 198-205.

8. Díaz WJV, Ticona GMC, Copa EGC, Mamani CL, Villanueva ERAG. Factores que influyen en la adherencia a la suplementación con SF en el embarazo, Tacna 2015. Rev Médica Basadrina. 2018; 12(1): 16-22.

9. Page MJ, McKenzie JE, Bossuyt PM, Boutron I, Hoffmann TC, Mulrow CD, et al. The PRISMA 2020 statement: an updated guideline for reporting systematic reviews. BMJ. 2021; 372: n71.

10. The Cochrane Collaboration. Review Manager (RevMan) [Computer program]. Version 5.4. 2020.

11. Higgins JPT, Altman DG, Gøtzsche PC, Jüni P, Moher $\mathrm{D}$, Oxman $\mathrm{AD}$, et al. The Cochrane Collaboration's tool for assessing risk of bias in randomised trials. BMJ. 2011; 343: d5928.

12. Schünemann HJ, Oxman AD, Brozek J, Glasziou P, Jaeschke R, Vist GE, et al. Grading quality of evidence and strength of recommendations for diagnostic tests and strategies. BMJ. 2008; 336(7653): 1106-10.

13. Ortiz R, Toblli JE, Romero JD, Monterrosa B, Frer C, Macagno E, et al. Efficacy and safety of oral iron(III) polymaltose complex versus ferrous sulfate in pregnant women with iron-deficiency anemia: a multicenter, randomized, controlled study. J Matern Fetal Neonatal Med. 2011; 24(11): 1347-52. 
14. Saha L, Pandhi P, Gopalan S, Malhotra S, Saha PK. Comparison of efficacy, tolerability, and cost of iron polymaltose complex with ferrous sulphate in the treatment of iron deficiency anemia in pregnant women. MedGenMed. 2007; 9(1): 1.

15. CADHT. Oral Iron for Anemia: A Review of the Clinical Effectiveness, Cost-effectiveness and Guidelines [Internet]. Ottawa (ON): Canadian Agency for Drugs and Technologies in Health; 2016 [citado el 28 de mayo de 2021]. (CADTH Rapid Response Reports). Disponible en: http://www.ncbi. nlm.nih.gov/books/NBK343969/

16. Reveiz L, Gyte GM, Cuervo LG, Casasbuenas A. Treatments for iron-deficiency anaemia in pregnancy. Cochrane Database Syst Rev. 2011; (10): CD003094.

17. Sloan NL, Jordan E, Winikoff B. Effects of Iron Supplementation on Maternal Hematologic Status in Pregnancy. Am J Public Health. 2002; 92(2): 28893.

18. Jacobs P, Fransman D, Coghlan P. Comparative bioavailability of ferric polymaltose and ferrous sulphate in iron-deficient blood donors. J Clin Apheresis. 1993; 8(2): 89-95.

19. Mei Z, Cogswell ME, Parvanta I, Lynch S, Beard JL, Stoltzfus RJ, et al. Hemoglobin and ferritin are currently the most efficient indicators of population response to iron interventions: an analysis of nine randomized controlled trials. J Nutr. 2005; 135(8): 1974-80.
20.Pena-Rosas JP, Viteri FE. Effects of routine oral iron supplementation with or without folic acid for women during pregnancy. Cochrane Database Syst Rev. 2006; (3): CD004736.

21. Toblli JE, Brignoli R. Iron(III)-hydroxide polymaltose complex in iron deficiency anemia / review and meta-analysis. Arzneimittelforschung. 2007; 57(6A): 431-8.

22. Melamed N, Ben-Haroush A, Kaplan B, Yogev Y. Iron supplementation in pregnancy--does the preparation matter? Arch Gynecol Obstet. 2007; 276(6): 601-4.

23. Souza AI de, Batista Filho M, Bresani CC, Ferreira LOC, Figueiroa JN. Adherence and side effects of three ferrous sulfate treatment regimens on anemic pregnant women in clinical trials. Cad Saúde Pública. 2009; 25: 1225-33.

24. Desta M, Kassie B, Chanie H, Mulugeta H, Yirga T, Temesgen $\mathrm{H}$, et al. Adherence of iron and folic acid supplementation and determinants among pregnant women in Ethiopia: a systematic review and metaanalysis. Reprod Health. 2019; 16(1): 182.

25. Sendeku FW, Azeze GG, Fenta SL. Adherence to iron-folic acid supplementation among pregnant women in Ethiopia: a systematic review and metaanalysis. BMC Pregnancy Childbirth. 2020; 20(1): 138. 